\title{
Serum microRNA-17 functions as a prognostic biomarker in osteosarcoma
}

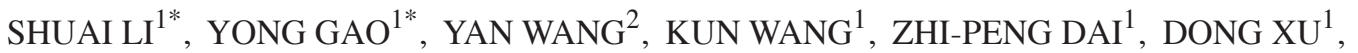 \\ WEI LIU ${ }^{1}$, ZHI-LIANG LI ${ }^{1}$, ZHENG-DONG ZHANG ${ }^{1}$, SHU-HUA YANG $^{1}$ and CAO YANG ${ }^{1}$ \\ ${ }^{1}$ Department of Orthopedics, Union Hospital, Tongji Medical College, Huazhong University of Science and Technology, \\ Wuhan, Hubei 430022; ${ }^{2}$ Section of Physical Education, China University of Geosciences, \\ Wuhan, Hubei 430074, P.R. China
}

Received January 24, 2015; Accepted March 15, 2016

DOI: $10.3892 / 01.2016 .5362$

\begin{abstract}
MicroRNAs (miRNAs/miRs) are a class of small noncoding RNA molecules that have important roles in regulating the expression of target genes associated with the development and progression of cancer. The majority of miRNAs are expressed in a highly tissue- and region-specific manner, and released into the bloodstream as a consequences of different diseases. Furthermore, altered levels of miRNAs have been observed in several diseases, including cancer. In the present study, reverse transcription-quantitative polymerase chain reaction (RT-qPCR) demonstrated that circulating miR-17 levels were significantly upregulated in patients with osteosarcoma (OS) compared with healthy subjects. RT-qPCR also revealed that high levels of circulating miR-17 expression were inversely correlated with phosphatase and tensin homolog expression, which was identified as a target gene of miR-17 in OS tissues. Furthermore, the overall survival of patients with OS was shorter in those with high miR-17 expression compared with moderate and low expression. Taken together, these findings indicate that miR-17 may function as a useful diagnostic and prognosis biomarker or therapeutic target of OS.
\end{abstract}

\section{Introduction}

Osteosarcoma (OS) is an aggressive disease, and is the most common type of bone tumor in children and adolescents globally (1). The tumor predominantly occurs near regions that produce osteoid. The prognosis of patients with OS

Correspondence to: Professor Cao Yang, Department of Orthopedics, Union Hospital, Tongji Medical College, Huazhong University of Science and Technology, 1277 Jiefang Road, Wuhan, Hubei 430022, P.R. China

E-mail: yangcaomd@sina.com

*Contributed equally

Key words: osteosarcoma, microRNA-17, phosphatase and tensin homolog, serum, biomarkers, prognosis is poor due to its high rate of metastasis and chemoresistance (2). Approximately one-third of patients diagnosed with localized OS experience relapse or progressive illness, and the mean survival time of patients with OS recurrence is $<1$ year (3). Prior to the emergence of effective chemotherapy, 2-year overall survival rates following surgical resection and/or radiotherapy were $15-20 \%(4,5)$. Further advances in therapeutic strategies for OS, including wide tumor excision, neoadjuvant and adjuvant chemotherapy, and radiotherapy, have resulted in significantly improved clinical outcomes and long-term survival rates $(2,6-9)$. However, $>30 \%$ of patients with OS survive for $<5$ years and succumb due to pulmonary metastases following diagnosis $(10,11)$. Therefore, it is critical to identify OS effector molecules, novel therapeutic strategies, and signaling pathways regulating OS growth and metastasis. Furthermore, there is an ongoing need for a more thorough understanding of the genetic and molecular mechanisms of OS development and metastasis.

Emerging evidence in the research area of epigenetics suggests that OS is caused by the accumulation of genetic and epigenetic alterations (12). MicroRNAs (miRNAs) are highly conserved, noncoding, small (22 nucleotides in length) RNAs that have a critical role in regulating post-transcriptional gene expression $(13,14)$. Aberrant miRNA expression affects the initiation and progression of carcinogenesis, and is associates with abnormal cell proliferation, differentiation, growth, apoptosis and invasion (15). Recent advances in epigenetics have revealed that miRNA dysregulation frequently occurs in various types of neoplasm, including gastric, colon, lung and prostate cancer, as well as OS (16-20). The focus of a vast number of studies on determining the expression of miRNAs in clinical samples has identified an association between miRNA function and oncogenesis or tumor suppressor genes, which was dependent on the function of the miRNA target genes $(21,22)$.

In recent decades, the majority of studies have focused on miRNA expression in cell lines and tissues; these miRNAs have also been identified in circulation as diagnostic and prognostic biomarkers (23). Further studies have shown that the circulating miRNA profile reflects disease states, particularly in cancer $(24,25)$. However, the association between miRNA profiles in circulation and in tumor tissues remains unclear. 
In our previous study (20), it was found that miR-17 levels were marginally elevated in OS tissues and cell lines. The study also observed that downregulation of miR-17 expression in OS cells, which leads to increased phosphatase and tensin homolog (PTEN) expression, resulted in the suppression of OS cell proliferation, migration and invasion. However, expression of miR-17 in the circulation has not been predicted or experimentally confirmed, and the expression levels and patterns of miR-17 in the circulation of patients with OS have not been well established. Therefore, the present study detected miR-17 expression in OS cell lines and serum samples. miR-17 expression was upregulated in the serum and positively correlated with expression levels in the OS specimens. These results suggest that miR-17 may be a promising prognostic biomarker of OS.

\section{Materials and methods}

Cell lines, blood samples and OS specimens. Human osteoblast cell line hFOB 1.19, and OS cell lines U2OS, Saos-2, MG-63 and MNNG/HOS were purchased from The Cell Bank of Type Culture Collection of Chinese Academy of Sciences, Shanghai Institute of Cell Biology, Chinese Academy of Sciences (Shanghai, China). Human osteosarcoma cell lines MNNG/HOS, U2OS, Saos-2 and MG-63 were cultured in minimal essential medium, Dulbecco's modified Eagle's medium (DMEM), RPMI 1640 and DMEM, respectively, supplemented with $10 \%$ fetal bovine serum (FBS), $100 \mathrm{mg} / \mathrm{ml}$ streptomycin and $100 \mathrm{IU} / \mathrm{ml}$ penicillin, at $37^{\circ} \mathrm{C}$ with $5 \% \mathrm{CO}_{2}$. The human osteoblast hFOB 1.19 cell line was maintained in DMEM/F12 medium supplemented with 10\% FBS, 100 $\mathrm{mg} / \mathrm{ml}$ streptomycin and $100 \mathrm{IU} / \mathrm{ml}$ penicillin, at $37^{\circ} \mathrm{C}$ with $5 \% \mathrm{CO}_{2}$. The cells were serum-starved for $12 \mathrm{~h}$ prior to each experiment.

The present study included 46 patients with OS and 46 matched normal tumor-free patients treated in the Department of Orthopedics, Union Hospital, Tongji Medical College, Huazhong University of Science and Technology (Wuhan, China) between March 2008 and March 2011. The patient cohort consisted of 27 males and 19 females, with an age at diagnosis that ranged from 9 to 48 years and a mean age of 19.6 years. Inclusion criteria were a diagnosis of osteosarcoma based on the results of imaging studies and biopsies, while exclusion criteria included the presence of any other type of neoplasm, liver and kidney dysfunction, and a post-operative pathological diagnosis of osteosarcoma. Peripheral blood samples $(15 \mathrm{ml})$ were obtained from the OS patients and matched normal tumor-free patients. The samples were centrifuged at $978 \times \mathrm{g}$ for $10 \mathrm{~min}$ at room temperature, serum was extracted and stored immediately in liquid nitrogen until use.

OS and matched normal non-tumor tissues $(n=46)$ were collected from the patients with OS. All the tissues were resected at the time of surgery (including amputation and limb-sparing surgery) and immediately stored in liquid nitrogen until use. The present study was approved by the Ethics Committee of Union Hospital, Tongji Medical College, Huazhong University of Science and Technology.

RNA isolation and reverse transcription-quantitative polymerase chain reaction $(R T-q P C R)$. Total miRNA was obtained from all cultured cells, serum samples and tissues using RNeasy Mini and miRNeasy Mini kits (Qiagen, Inc., Valencia, CA, USA). miR-17 expression was quantified by performing RT-qPCR using TaqMan MicroRNA Assay kits (catalog no. 217004; Applied Biosystems; Thermo Fisher Scientific, Inc., Waltham, MA, USA) on a LightCycler 480 System II (Roche Diagnostics, Rotkreuz, Switzerland). The primer sequences were as follows: Forward, 5'-CCAGGACCAGAGGAAACCT-3' and reverse, 5'-GCTAGCCTCTGG ATTTGA-3' for PTEN; U6 forward, 5'-CTCGCTTCGGCA GCACA-3' and reverse, 5'-AACGCTTCACGAATTTGC GT-3'; miR-17 forward, 5'-TGCTTACAGTGCAGGTAG-3' and reverse, 5'-GAACATGTCTGCGTATCTC-3'; and forward, 5'-ATGTCGTGGAGTCTACTGGC-3' and reverse, 5'-TGA CCTTGCCCACAGCCTTG-3' for GAPDH. The PCR conditions were as follows: $50^{\circ} \mathrm{C}$ for $2 \mathrm{~min}, 95^{\circ} \mathrm{C}$ for $10 \mathrm{~min}, 95^{\circ} \mathrm{C}$ for $30 \mathrm{sec}$ and $60^{\circ} \mathrm{C}$ for $30 \mathrm{sec}$, for 40 cycles. PTEN expression was detected using SYBR Premix Ex Taq II (Takara Bio, Inc., Otsu, Japan). The RT-qPCR data was evaluated using the $2^{-\Delta \Delta \mathrm{Ct}}$ method (26) relative to GAPDH for mRNA or U6 small nuclear RNA for miRNA. Each PCR experiment was conducted in triplicate.

Statistical analysis. Data are presented as mean \pm standard deviation and were analyzed using SPSS software (version 16.0; SPSS, Inc., Chicago, IL, USA). The correlation between miR-17/PTEN expression levels and prognosis was analyzed by Kaplan-Meier survival analysis using GraphPad Prism 6 software (GraphPad Software, Inc., San Diego, CA, USA). Analysis of variance (followed by Gabriel's procedure or a two-tailed Student's t-test were used to examine the data between $>2$ or 2 variables, respectively, and Spearman's correlation analysis was performed to evaluate the association between miR-17 and PTEN expression levels. $\mathrm{P}<0.05$ was used to indicate a statistically significant difference.

\section{Results}

Expression of miR-17 is increased in OS cell lines, serum samples and tissues. To investigate the potential significance of miR-17 in OS development and progression, the present study evaluated the expression of miR-17 in a human osteoblast cell line, and OS cell lines, serum samples and tissues using RT-qPCR. miR-17 expression levels were significantly upregulated in all four OS cell lines, U2OS, Saos-2 (P<0.05), MG-63 and MNNG/HOS $(\mathrm{P}<0.01)$, compared with the hFOB 1.19 human osteoblast cell line (Fig. 1A). The expression of miR-17 was also significantly increased in serum samples of patients with OS compared with normal controls subjects $(\mathrm{P}<0.05$; Fig. 1B). Furthermore, the expression of miR-17 was significantly increased in OS tissues compared with matched normal tissues $(\mathrm{P}<0.05$; Fig. 1C).

Correlation between miR-17 and PTEN expression in patients with OS. Previous studies demonstrated that PTEN is a target gene of miR-17 and its expression appears to be inversely correlated with miR-17 expression levels in OS tissues $(27,28)$. In the present study, PTEN expression was significantly decreased in human OS tissues compared with in matched normal tissues $(\mathrm{P}<0.01)$, indicating that downregulation of 
Table I. Comparison of the clinical characteristics of 46 OS patients with PTEN tissue expression and circulating miR-17 expression.

\begin{tabular}{|c|c|c|c|c|c|c|c|c|}
\hline \multirow[b]{2}{*}{ Characteristic } & \multicolumn{4}{|c|}{ PTEN expression in OS tissue } & \multicolumn{4}{|c|}{ miR-17 expression in serum } \\
\hline & High & Moderate & Low & P-value & High & Moderate & Low & P-value \\
\hline Total, n (\%) & $12(26.1)$ & $12(26.1)$ & $22(47.8)$ & 0.523 & $25(54.3)$ & $12(26.1)$ & $9(19.6)$ & 0.546 \\
\hline \multicolumn{9}{|c|}{ Age at diagnosis, years } \\
\hline Mean & 21.9 & 24.3 & 16.7 & & 15.2 & 23.8 & 24.7 & \\
\hline Range & $13-42$ & $12-48$ & $9-27$ & 0.673 & $9-31$ & $14-40$ & $13-48$ & 0.615 \\
\hline \multicolumn{9}{|l|}{ Gender, n (\%) } \\
\hline Male & $7(58.3)$ & $6(50.0)$ & $14(63.4)$ & & $15(60.0)$ & $7(58.3)$ & $6(66.7)$ & \\
\hline Female & $5(41.7)$ & $6(50.0)$ & 8 (32.6) & 0.632 & $10(40.0)$ & $5(41.7)$ & $3(33.3)$ & 0.754 \\
\hline \multicolumn{9}{|c|}{ Prognosis, n (\%) } \\
\hline Survived & $7(58.3)$ & $8(66.7)$ & $9(40.9)$ & 0.045 & $10(40.0)$ & $9(75.0)$ & $5(55.6)$ & 0.042 \\
\hline Deceased & $5(41.7)$ & $4(33.3)$ & $13(59.1)$ & 0.032 & $15(60.0)$ & $3(25.0)$ & $4(44.4)$ & 0.036 \\
\hline
\end{tabular}

OS, osteosarcoma; PTEN; phosphatase and tensin homolog; miR, microRNA.

A

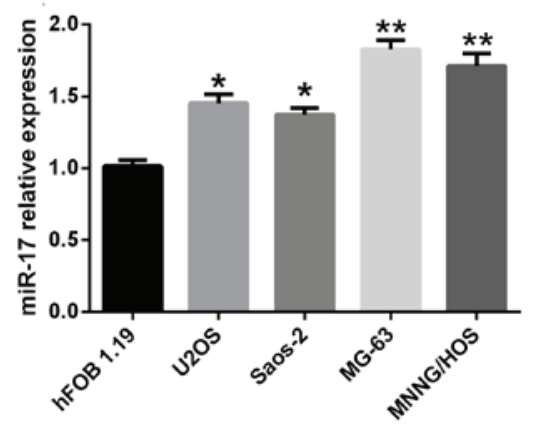

B

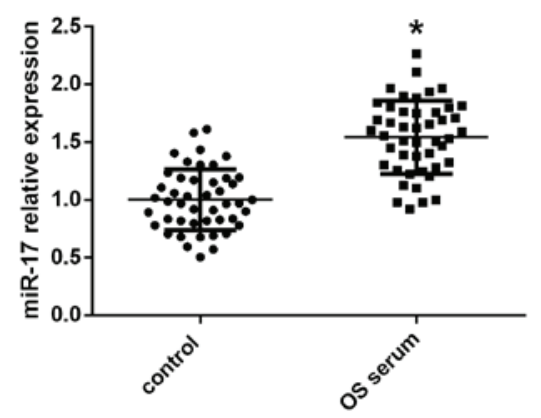

C

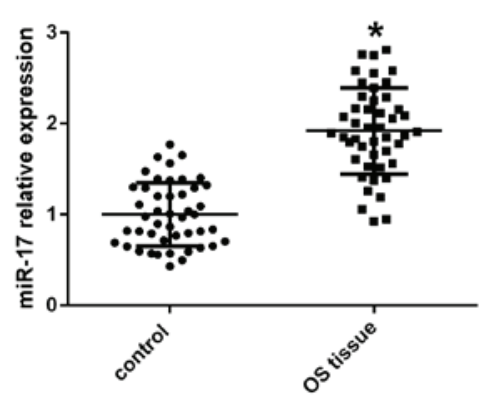

Figure 1. Expression of miR-17 is increased in OS cell lines, serum samples and tissues. miR-17 expression was significantly increased in (A) U2OS, MG-63, Saos-2 and MNNG/HOS cells lines compared with the hFOB 1.19 osteoblast cell line $(\mathrm{P}=0.0218, \mathrm{P}=0.0286, \mathrm{P}=0.00852$ and $\mathrm{P}=0.00937$ vs. $\mathrm{hFOB} 1.19$ cells $)$; (B) OS serum samples compared with normal serum samples $(\mathrm{P}=0.0254)$; and $(\mathrm{C}) \mathrm{OS}$ tissues compared with paired normal tissues $(\mathrm{P}=0.0370)$. Data are presented as mean \pm standard deviation. ${ }^{*} \mathrm{P}<0.05,{ }^{* *} \mathrm{P}<0.01$ vs. hFOB 1.19 cells or the control group. miR, microRNA; OS, osteosarcoma..

PTEN may have a significant role in OS development and progression (Fig. 2A). To evaluate the association between circulating miR-17 and PTEN expression levels, Spearman's correlation analysis was performed, demonstrating that serum miR-17 expression is strongly inversely correlated with PTEN expression in OS tissue ( $\mathrm{P}=0.0002$; Fig. 2B).

Upregulation of miR-17 expression and downregulation of PTEN expression are correlated with poor survival in patients with OS. To further determine the potential roles of miR-17 in OS development and progression, the present study analyzed the correlations between miR-17 expression in circulation and PTEN expression in OS tissues, and clinical features of patients with OS. The expression levels of miR-17 in serum and PTEN in OS tissues were analyzed in the cohort of 46 patients with OS, and divided into the following three groups: Low expression (score 1), moderate expression (score 2) and high expression (score 3). The results revealed that high expression of miR-17 in circulation was strongly associated with poor prognosis in patients with OS, while overexpression of PTEN in OS tissues was significantly correlated with good prognosis in patients with OS $(\mathrm{P}<0.05$; Table I). As indicated in Table I, the expression levels of miR-17 were correlated with incidence at an early age $(\mathrm{P}>0.05)$.

Furthermore, Kaplan-Meier analysis demonstrated that the prognosis of patients with OS is significantly worse in those with high serum miR-17 expression compared with those exhibiting moderate and low miR-17 expression $(\mathrm{P}=0.0037$; Fig. 3A). By contrast, patients with high PTEN tissue expression exhibited a significantly better prognosis than those with moderate and low PTEN expression ( $\mathrm{P}=0.0187$; Fig. $3 \mathrm{~B})$. Furthermore, patients were divided into two outcome groups: Survival group (survived for $>5$ years) and non-survival group (survived for $<5$ years). It was observed that patients in the survival group (24 patients, 52.2\%) exhibited significantly lower miR-17 expression scores compared with patients in the non-survival group (22 patients, $47.8 \%$; $\mathrm{P}=0.0026$ ). By contrast, patients in the survival group had higher PTEN expression scores than patients in the non-survival group $(\mathrm{P}=0.0002)$. The mean miR-17 and PTEN expression score 
A

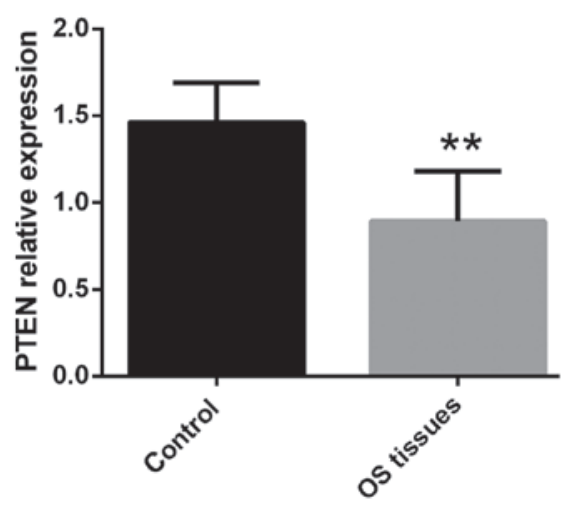

B

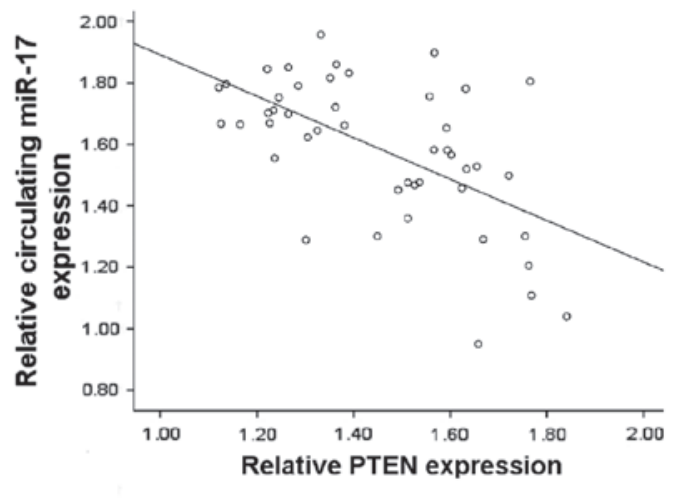

Figure 2. PTEN expression and correlation between miR-17 and PTEN expression in patients with OS. (A) Relative PTEN mRNA expression was downregulated in OS tissues compared with normal tissues $(\mathrm{P}=0.00823)$. (B) Inverse associations were observed between circulating miR-17 expression levels and PTEN expression level in OS tissue samples (Spearman's correlation analysis: $\mathrm{r}=-0.5297, \mathrm{P}=0.0002$ ). Data are presented as mean \pm standard deviation. ${ }^{* *} \mathrm{P}<0.01 \mathrm{vs}$. the control group. PTEN, phosphatase and tensin homolog; OS, osteosarcoma; miR, microRNA.

A

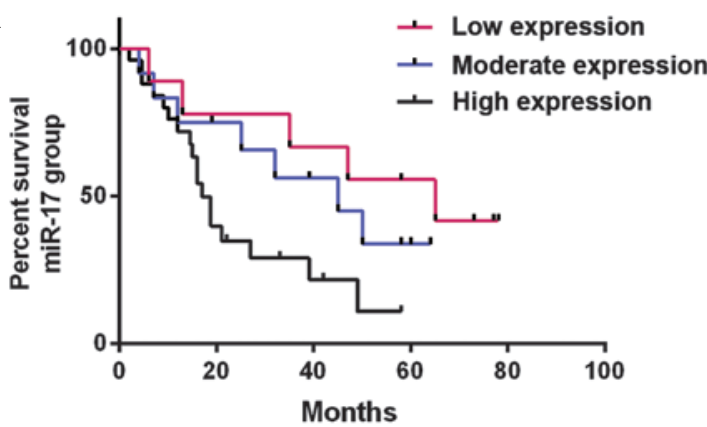

C

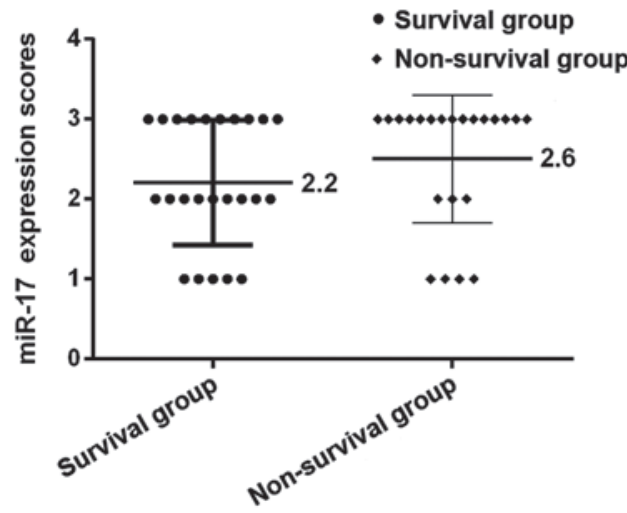

B

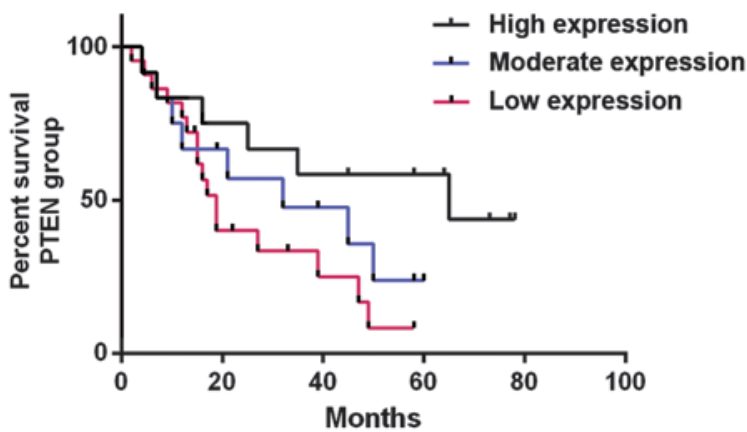

D

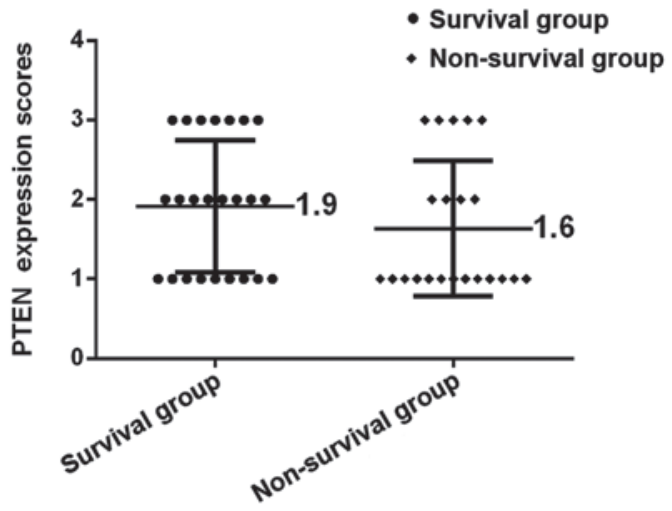

Figure 3. Upregulation of miR-17 expression and downregulation of PTEN expression are correlated with poor survival of patients with osteosarcoma (OS), Kaplan-Meier survival curves of patients with OS were divided 3 groups depending on high, moderate and low expression of miR-17 and PTEN. (A) Survival curve showing that the overall survival of OS patients with high expression of miR-17 was significantly shorter than patients with moderate and low miR-17 expression ( $\mathrm{P}=0.0037)$. (B) Survival curve showing that the overall survival of OS patients with high expression of PTEN was significantly longer than those patients with moderate and low PTEN expression $(\mathrm{P}=0.0187)$. (C) miR-17 expression scores of OS patients in the non-survival group were significantly higher than in patients in the survival group $(\mathrm{P}=0.0026)$. (D) PTEN expression scores of OS patients in the survival group were significantly higher than in patients in the non-survival group $(\mathrm{P}=0.002)$. miR, microRNA; PTEN, phosphatase and tensin homolog.

ranges in two groups were 2.2-2.6 (Fig. 3C) and 1.9-1.6 (Fig. 3D), respectively.

\section{Discussion}

Emerging studies have indicated that miRNAs are involved in several processes, including cell apoptosis, proliferation, metastasis and differentiation, by regulating the expression of multiple target genes $(14,15)$. Hence, exploring the profiles of miRNAs and their target genes involved in tumorigenesis may promote understanding of the underlying mechanisms of patients with human malignancies, and provide valuable insight for the early diagnosis and treatment of such diseases $(20-22,24)$. Results from previous studies have continually demonstrated that the presence of miRNAs in the circulation and in bodily fluid may function as promising diagnostic and prognostic biomarkers in certain diseases, including human malignancies (23-25,29). 
In the present study, the expression of miR-17 in serum samples from patients with OS was compared with paired serum samples of healthy control subjects. This analysis revealed that the expression of miR-17 in circulation was significantly upregulated in OS compared with the normal controls. The current study also observed increased expression of miR-17 in OS tissues compared with normal tissues. This is consistent with a previous study, which identified that miR-17 expression is upregulated in OS cell lines compared with a matched human osteoblast cell line (20). Simiarly, Minami et al demonstrated that the overexpression of miR-17 promotes cell growth in synovial sarcoma (27). Our results identified PTEN as a miR-17 target gene that affects OS cell proliferation, colony formation, migration and invasion (20). These results are consistent with a previous report that PTEN is a direct target of miR-17 in glioblastoma cells (30). Our previous study demonstrated that downregulation of miR-17 leads to the upregulation of PTEN expression in OS cells, which results in the inhibition of OS cell growth and metastasis (20). Similarly, a previous study concerning PTEN expression levels in OS tissues revealed a the positive correlation between PTEN expression and the degree of differentiation (31).

Initially identified in 1997, the PTEN protein is considered an important tumor suppressor that inhibits tumor development, invasion, angiogenesis and metastasis (32). PTEN predominantly functions as a tumor suppressor gene through the phosphoinositide 3-kinase (PI3K)/PTEN/AKT signaling pathway. It has been reported that the PI3K/PTEN/AKT signaling pathway is frequently deregulated during tumorigenesis (33). Considering the importance of the PI3K/PTEN/AKT signaling pathway in regulating numerous cellular behaviors, including cell proliferation and survival, it is unsurprising that the PTEN gene undergoes inactivation or loss of function in several types of human tumor (34). As a tumor suppressor gene, downregulation of PTEN in certain types of malignant cancer activates AKT, promoting cell proliferation, survival, migration and angiogenesis (35). Immunohistochemical characterization of OS tumor tissues suggest that PTEN expression is downregulated in a high percentage of OS cases (36). Furthermore, the present study results suggest that miR-17 modulates PTEN expression and that miR-17 expression is downregulated in OS serum, which reveals the involvement of miR-17 in the pathological process of OS.

In conclusion, the current results demonstrated that downregulation of miR-17 decreases OS cell survival, and enhanced miR-17 expression resulted in the downregulation of PTEN expression in OS. Taken together, the current results suggest, for the first time, that miR-17 in serum may function as a diagnostic biomarker and prognostic factor in the progression of OS.

\section{References}

1. Marina N, Gebhardt M, Teot L and Gorlick R: Biology and therapeutic advances for pediatric osteosarcoma. Oncologist 9: 422-441, 2004

2. Ferguson WS and Goorin AM: Current treatment of osteosarcoma. Cancer Investigation 19: 292-315, 2001.

3. Schwartz CL, Gorlick R, Teot L, Krailo M, Chen Z, Goorin A, Grier HE, Bernstein ML and Meyers P; Children's Oncology Group: Multiple drug resistance in osteogenic sarcoma: INT0133 from the children's oncology group. J Clin Oncol 25: 2057-2062, 2007.
4. Marcove RC, Miké V, Hajek JV, Levin AG and Hutter RV: Osteogenic sarcoma under the age of twenty-one. A review of one hundred and forty-five operative cases. J Bone Joint Surg Am 52: 411-423, 1970.

5. Friedman MA and Carter SK: The therapy of osteogenic sarcoma: Current status and thoughts for the future. J Surg Oncol 4: 482-510, 1972.

6. Bernthal NM, Federman N, Eilber FR, Nelson SD, Eckardt JJ, Eilber FC and Tap WD: Long-term results ( $>25$ years) of a randomized, prospective clinical trial evaluating chemotherapy in patients with high-grade, operable osteosarcoma. Cancer 118: 5888-5893, 2012

7. Yang J and Zhang W: New molecular insights into osteosarcoma targeted therapy. Curr Opin Oncol 25: 398-406, 2013.

8. Bruland OS and Pihl A: On the current management of osteosarcoma. A critical evaluation and a proposal for a modified treatment strategy. Eur J Cancer 33: 1725-1731, 1997.

9. Amankwah EK, Conley AP and Reed DR: Epidemiology and therapies for metastatic sarcoma. Clin Epidemiol 5: 147-162, 2013.

10. Rainusso N, Wang LL and Yustein JT: The adolescent and young adult with cancer: State of the art-bone tumors. Curr Oncol Rep 15: 296-307, 2013.

11. Ottaviani G and Jaffe N: The epidemiology of osteosarcoma. Cancer Treat Res 152: 3-13, 2009.

12. Cote GM and Choy E: Role of epigenetic modulation for the treatment of sarcoma. Curr Treat Options Oncol 14: 454-464, 2013.

13. Zamore PD and Haley B: Ribo-gnome: The big world of small RNAs. Science 309: 1519-1524, 2005.

14. Ambros V: The functions of animal microRNAs. Nature 431: 350-355, 2004.

15. Cho WC: OncomiRs: The discovery and progress of microRNAs in cancers. Mol Cancer 6: 60, 2007.

16. Kim JG, Kim TO, Bae JH, Shim JW, Kang MJ, Yang K, Ting AH and Yi JM: Epigenetically regulated MIR941 and MIR1247 target gastric cancer cell growth and migration. Epigenetics 9: 1018-1030, 2014.

17. Fang L, Li H, Wang L, Hu J, Jin T, Wang J and Yang BB: MicroRNA-17-5p promotes chemotherapeutic drug resistance and tumour metastasis of colorectal cancer by repressing PTEN expression. Oncotarget 5: 2974-2987, 2014.

18. Okudela K, Tateishi Y, Umeda S, Mitsui H, Suzuki T, Saito Y, Woo T, Tajiri M, Masuda M, Miyagi Y and Ohashi K: Allelic imbalance in the miR-31 host gene locus in lung cancer-its potential role in carcinogenesis. PLoS One 9: e100581, 2014.

19. Jin M, Zhang T, Liu C, Badeaux MA, Liu B, Liu R, Jeter C, Chen X, Vlassov AV and Tang DG: MicroRNA-128 suppresses prostate cancer by inhibiting BMI-1 to inhibit tumor-initiating cells. Cancer Res 74: 4183-4195, 2014.

20. Gao Y, Luo LH, Li S and Yang C: miR-17 inhibitor suppressed osteosarcoma tumor growth and metastasis via increasing PTEN expression. Biochem Biophys Res Commun 444: 230-234, 2014.

21. Browne G, Taipaleenmäki H, Stein GS, Stein JL and Lian JB: MicroRNAs in the control of metastatic bone disease. Trends Endocrinol Metab 25: 320-327, 2014.

22. Mulrane L, McGee SF, Gallagher WM and O'Connor DP: miRNA dysregulation in breast cancer. Cancer Res 73: 6554-6562, 2013.

23. Bihrer V, Friedrich-Rust M, Kronenberger B, Forestier N, Haupenthal J, Shi Y, Peveling-Oberhag J, Radeke HH, Sarrazin C, Herrmann E, et al: Serum miR-122 as a biomarker of necroinflammation in patients with chronic hepatitis $\mathrm{C}$ virus infection. Am J Gastroenterol 106: 1663-1669, 2011.

24. Bryant RJ, Pawlowski T, Catto JW, Marsden G, Vessella RL, Rhees B, Kuslich C, Visakorpi T and Hamdy FC: Changes in circulating microRNA levels associated with prostate cancer. $\mathrm{Br}$ J Cancer 106: 768-774, 2012 .

25. Joosse SA, Müller V, Steinbach B, Pantel K and Schwarzenbach H: Circulating cell-free cancer-testis MAGE-A RNA, BORIS RNA, let-7b and miR-202 in the blood of patients with breast cancer and benign breast diseases. Br J Cancer 111: 909-917, 2014.

26. Livak KJ and Schmittgen TD: Analysis of relative gene expression data using real-time quantitative PCR and the 2(-Delta Delta C(T)) Method. Methods 25: 402-408, 2001.

27. Minami Y, Kohsaka S, Tsuda M, Yachi K, Hatori N, Tanino M, Kimura T, Nishihara H, Minami A, Iwasaki $\mathrm{N}$ and Tanaka S: SS18-SSX-regulated miR-17 promotes tumor growth of synovial sarcoma by inhibiting p21WAF1/CIP1. Cancer Sci 105: 1152-1159, 2014

28. Li X, Yang H, Tian Q, Liu Y and Weng Y: Upregulation of microRNA-17-92 cluster associates with tumor progression and prognosis in osteosarcoma. Neoplasma 61: 453-460, 2014. 
29. Zampetaki A, Kiechl S, Drozdov I, Willeit P, Mayr U, Prokopi M, Mayr A, Weger S, Oberhollenzer F, Bonora E, et al: Plasma microRNA profiling reveals loss of endothelial mir-126 and other microRNAs in type 2 diabetes. Circ Res 107: 810-817, 2010.

30. Li H and Yang BB: Stress response of glioblastoma cells mediated by miR-17-5p targeting PTEN and the passenger strand miR-17-3p targeting MDM2. Oncotarget 3: 1653-1668, 2012.

31. Wang Y, Chen A, Guo F and Xia Y: Expression and clinical significance of PTEN protein in osteosarcoma. Chinese-German J Clin Oncol 5: 296-299, 2008.

32. Li J, Yen C, Liaw D, Podsypanina K, Bose S, Wang SI, Puc J, Miliaresis C, Rodgers L, McCombie R, et al: PTEN, a putative protein tyrosine phosphatase gene mutated in human brain, breast and prostate cancer. Science 275: 1943-1947, 1997.
33. Di Cristofano A and Pandolfi PP: The multiple roles of PTEN in tumor suppression. Cell 100: 387-390, 2000.

34. Naguib A and Trotman LC: PTEN plasticity: How the taming of a lethal gene can go too far. Trends Cell Biol 23: 374-379, 2013.

35. Seront E, Pinto A, Bouzin C, Bertrand L, Machiels JP and Feron O: PTEN deficiency is associated with reduced sensitivity to mTOR inhibitor in human bladder cancer through the unhampered feedback loop driving PI3K/Akt activation. Br J Cancer 109: 1586-1592, 2013.

36. Levine RA, Forest T and Smith C: Tumor suppressor PTEN is mutated in canine osteosarcoma cell lines and tumors. Vet Pathol 39: 372-378, 2002. 